\title{
Unequal Error Recovery Scheme for Multimedia Streaming in Application-Level Multicast
}

\author{
Joonhyoung Lee, Youngha Jung, and Yoonsik Choe \\ Department of Electrical and Electronic Engineering, Yonsei University, \\ 134 Shinchon-dong Seodaemun-gu, Seoul, 120-749, South Korea \\ $\{$ jhlee019, crosscom, yschoe $\}$ @yonsei.ac.kr
}

\begin{abstract}
As a way of implementing multicast delivery, applicationlevel multicast (ALM) is known for its various advantages including high flexibility and easy deployment. However, one of its key limitations in achieving reliability and quality-of-service (QoS) for streaming services is the hierarchical error propagation caused by its tree structure. As a solution to this error propagation problem, we propose an unequal error recovery (UER) scheme where we apply different number of retransmissions to the different levels of the ALM tree. The error recovery scheme is implemented using reliable-UDP (RUDP). For each node of the ALM tree, the optimal number of the maximum retransmissions that minimizes the residual loss rate is obtained using Lagrange theory for a given delay constraint. Compared to the equal error recovery scheme where the equal number of maximum retransmissions is applied throughout the ALM tree, the proposed UER scheme shows up to $10 \%$ improvement in residual loss rate while maintaining acceptable delay overhead for real-time streaming applications.
\end{abstract}

Keywords: ALM, multicast, multimedia streaming, error recovery.

\section{Introduction}

Multicast delivery has been the preferred choice for the middle ground application between point-to-point unicast and large-audience broadcast 1]. In implementing the multicast functionality, two approaches have been widely used: IP multicast and application-level multicast (ALM). While IP multicast can provide the best performance in terms of the bandwidth on physical links, it has several drawbacks hindering its wider deployment. These include slow pace of deployment and the difficulties in reliability, congestion control and flow control. On the contrary, ALM can provide higher flexibility in streaming applications by implementing the multicast functionalities at the end hosts connected to the network. That is why it started receiving much attraction recently [1].

In general, ALM protocol is comprised of a logical tree rooted at source, where all the nodes are hosts and each link in the tree represents a network path. In this hierarchical structure, packet loss occurred in any links can be propagated to all the descendant links. The packet losses accumulated at the lower part of the tree can then cause severe performance degrading to its leaf nodes. Also, 
leaf nodes can suffer from a large amount of packet loss even though the link connected to the leaf node itself has no packet loss at all. Therefore, packet loss occurred in each link in the ALM tree has different impacts depending on its level. For this reason, we can construct more efficient and reliable ALM tree by making the higher levels of the tree more robust than its lower levels.

In order to recover packet losses in the ALM tree, many approaches have been proposed including forward error correction (FEC) based on redundant parity packets 3.4 and the retransmission scheme based on feedback mechanism [5]. While all these approaches show good error recovery performances in each link of the ALM tree, they still show the inherent limitation caused by the hierarchical error propagation. Recently, a lateral error recovery (LER) scheme 2] was proposed to improve the error recovery performance of the ALM tree. In LER, a node recovers its losses by retransmission not from its ancestor node but from some nearby recovery neighbors of other planes, which contain independent ALM trees. However, the performance of LER scheme can still be improved by taking the hierarchical error propagation into account. While the retransmission scheme can improve the performance, it has the side effects of increasing end-toend delays. For this reason, simply applying the same error recovery scheme to all the levels in the ALM tree can result in the large delay overhead especially at the leaf nodes.

In this paper, we propose a unequal error recovery (UER) scheme based on reliable-UDP (RUDP) 7]. UER has been designed and developed with the tradeoff between performance and delay overhead in mind. In other words, high performance scheme is applied to the higher levels of the ALM tree and low performance scheme is applied to the lower levels. To do this, the effect of the configurable parameters of RUDP on the performance is first analyzed and one of the dominant parameters - maximum number of retransmission - is selected to model the performance of the error recovery scheme. Then, optimal solution for the number of retransmission that minimizes overall packet loss rate is obtained. The optimization is performed using Lagrange theory with delay penalty as a constraint. Compared to the uniform error recovery scheme (where same type of recovery scheme is applied to all the levels in the ALM tree), UER shows up to $10 \%$ improvement in residual loss rate while maintaining a similar delay penalty.

Although we use RUDP in our UER approach, the retransmission scheme can be replaced by any kind of error recovery scheme as long as it can provide a means to apply the unequal performances depending on the level of the ALM tree. Also, different kinds of error recovery schemes can be applied to the different levels of the ALM tree in order to further improve the performance.

The remainder of this paper is organized as follows. In Sect. 2, basic error characteristics of ALM are presented. In Sect. 3, the performance of retransmission scheme with RUDP is modeled using its configurable parameters and an optimal solution for the parameter is obtained for UER using Lagrange theory. Experimental results are shown in Sect. 4 followed by the conclusion in Sect. 5 . 


\section{Error Characteristics in ALM Tree}

Among various ALM tree construction algorithms, the binary tree is selected to construct an ALM tree in order to analyze the error characteristics. In Fig. 1] the packet 3 , which is lost between source $S$ and node $A$ becomes lost packets in all descendants. While the loss of packet 8 from node $B$ to node $C$ affects node $C$ only, the loss of packet 3 between source $S$ and node $A$ consequently means packet loss to the other six nodes. That is, the number of nodes affected by packet loss strongly depends on the level of the hierarchical ALM tree.

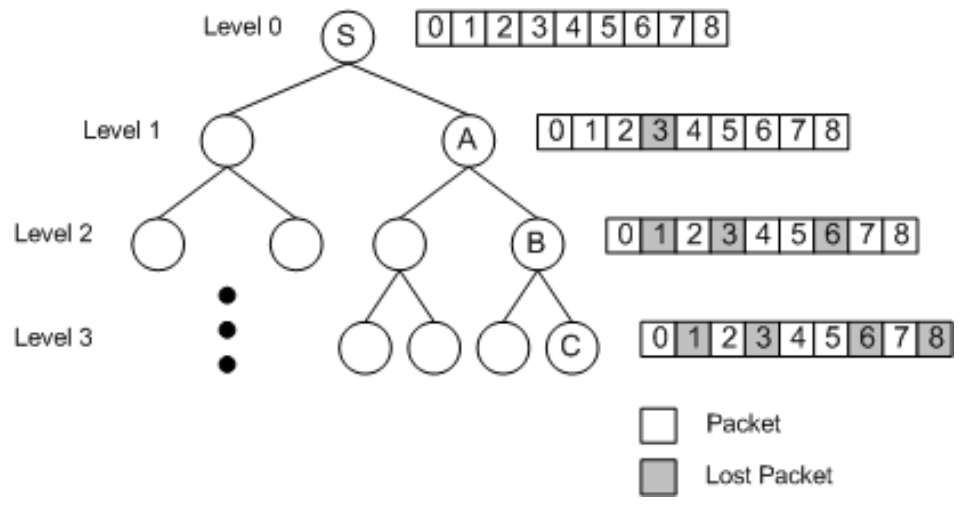

Fig. 1. Error propagation property through the link of the ALM tree

By considering the error propagation described above, decreasing packet losses in the higher level of the ALM tree can improve the performance of the ALM. While minimizing the packet loss rate in all levels of an ALM tree is the best solution, improvement of error recovery at all levels might cause the following side effects: 1) For FEC, increasing redundant packet reduces packet loss rate, while bandwidth efficiency is reduced. 2) In case of retransmission scheme, incrementing the number of retransmission accompanies additional delay in network path although it improves the error recovery performance. There is a strong possibility that these accumulated side effects in the ALM tree deteriorate the service quality of streaming service severely. For this reason, these side effects should be considered all together to optimize the performance of error recovery scheme.

\section{Unequal Error Recovery (UER) Scheme}

In this section we analyze the retransmission scheme and model its delay and packet loss rate in terms of the RUDP parameters. Based on the analysis, we find the optimal values of the parameter, the maximum number of retransmission, which minimizes the packet loss rate subject to the delay constraint. 


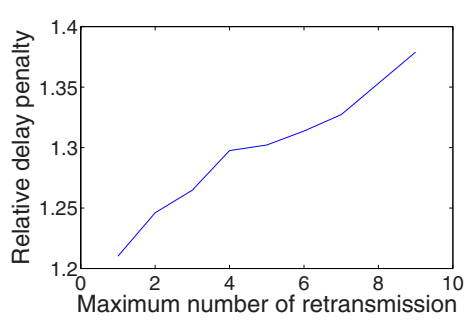

(a) RDP

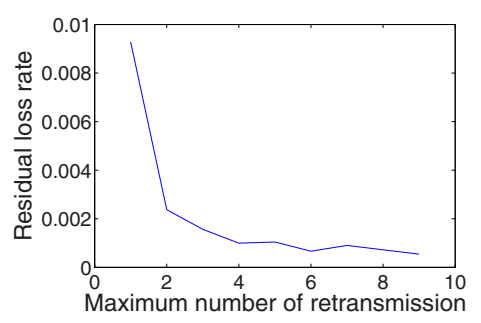

(b) RLR

Fig. 2. The performance of error recovery scheme in terms of the maximum number of retransmission

\subsection{Modeling the Performance of Retransmission Scheme}

Although TCP provides $100 \%$ reliability by means of retransmission, it is not suitable for real-time streaming application 2. UDP is widely used for real-time streaming application. However it does not provide any error recovery scheme at all. We use RUDP [7] as an error recovery scheme because it provides retransmission mechanism and high flexibility with various configurable parameters. It can also be easily and effectively applied to any real-time streaming applications and show acceptable performance.

Error recovery performance and delay of error recovery scheme are easily controlled by several parameters in RUDP. Because these parameters have tradeoff between error recovery performance and delay, we need to analyze the effects of these parameters on the performance of error recovery scheme. To analyze the performance, we use the following two performance measures.

- Relative Delay Penalty (RDP); it is defined as the ratio of the delay in the overlay to the delay in error-free delivery.

- Residual Loss Rate (RLR); it is defined as the overall packet loss rate for all packets

In order to measure the performance of RUDP in terms of each parameter, large amount of experiments are done with various combination of the parameters for various packet loss rates. These experiments show that the maximum number of retransmission, $N$, is the dominant parameter in terms of its impacts on the error recovery performance and delay characteristics. This is also backed up by the fact that setting all the other RUDP parameters (except $N$ ) as recommended by [7] does not show performance variations compared to setting them to the other values (this results are omitted due to the limit of this paper)

As an example, Fig. 2 shows the performance of error recovery scheme in terms of the maximum number of retransmission, $N$. We set the packet loss rate of links to $10 \%$ in this experiment. As it can be inferred from Fig. 2, RDP 
has a linear relationship to $N$ and RLR follows the inverse square curve of $\mathrm{N}$. Accordingly, RDP and RLR can be modeled as follows.

$$
\begin{gathered}
D_{i}=\alpha N_{i}+\beta . \\
R_{i}=\frac{\gamma}{N_{i}^{2}} .
\end{gathered}
$$

where $D_{i}$ and $R_{i}$ are RDP and RLR measured at link $i$, respectively, $N_{i}$ is the maximum number of retransmission, and $\alpha, \beta, \gamma$ are modeling coefficients.

\subsection{Optimal Unequal Error Recovery Scheme}

Using the basic models in (11) and (2), the performance of error recovery schemes along a single network path from a source to a leaf node can be expressed as follows.

$$
\begin{gathered}
D=\sum_{i=1}^{T} D_{i}=\sum_{i=1}^{T}\left(\alpha N_{i}+\beta\right) . \\
R=\sum_{i=1}^{T} w_{i} R_{i}=\sum_{i=1}^{T} \frac{w_{i} \gamma}{N_{i}^{2}} .
\end{gathered}
$$

where $D$ and $R$ are RDP and RLR measured along a single network path from the source to the leaf node, respectively, $T$ is the number of links and $w_{i}$ is the weighting factor that represents the number of descendants that are affected by the packet lost in the $i$ th link. Now we want to find the expression for the maximum number of retransmission for each level, $N_{1}^{*}, \ldots, N_{T}^{*}$, that minimize RLR in (4) while satisfying the condition such that the delay from the source to the leaf node must be equal to or lower than the delay constraint, $D_{\text {total }}$ :

$$
N_{1}^{*}, \ldots, N_{T}^{*}=\underset{\substack{N_{1}, \ldots, N_{T} \\ \sum_{i=1}^{T} D_{i} \leq D_{\text {total }}}}{\operatorname{argmin}} \sum_{i=1}^{T} \frac{w_{i} \gamma}{N_{i}^{2}} .
$$

Observe that, since we are minimizing a convex, differentiable function on a convex set, there is a unique solution that can be obtained using Lagrange theory 6. To do this, we define $\lambda$ and $\lambda^{*}$ as the Lagrange multiplier and its optimal value, respectively. Then the optimization problem in (5) can be rewritten in its equivalent (unconstrained) form:

$$
N_{1}^{*}, \ldots, N_{T}^{*}, \lambda^{*}=\underset{N_{1}, \ldots, N_{T}, \lambda}{\operatorname{argmin}} \sum_{i=1}^{T} \frac{w_{i} \gamma}{N_{i}^{2}}+\lambda\left[\sum_{i=1}^{T}\left(\alpha N_{i}+\beta\right)-D_{\text {total }}\right] .
$$

By setting partial derivatives to zero in (6) , we can obtain the following expression for the optimized value for the maximum number of retransmission at each level.

$$
N_{i}^{*}=\sqrt[3]{\frac{2 w_{i} \gamma}{\alpha}} \cdot \frac{D_{\text {total }}-T \beta}{\sum_{i=1}^{T} \sqrt[3]{2 \alpha^{2} w_{i} \beta}}
$$


The experiments using this optimal value in the ALM tree is explained in the next section.

\section{Experimental Results}

For the performance experiments, we generated a number of ALM trees using the NS-2 simulator [8]. Each node in the tree is connected to a router with $1 \mathrm{~ms}$ delay, and each router is connected to the other routers with $30 \mathrm{~ms}$ delay. For an ALM tree, the basic form of binary tree was chosen due to its simplicity for analysis. We used MPEG-4 compressed bitstream for streaming application and RTP as a streaming protocol.

Packets transmitted by multicast were randomly dropped in physical links. Packet loss rates were selected in accordance with LER [2]: with the probability of 0.95 the packet loss rate of a link is uniformly distributed between 0 and $1 \%$, and with the probability of 0.05 the packet loss rate is uniformly distributed between 5 and 10\%. Packets were divided into two types, such as data packets for video bitstream and control packets for control message. We assumed that control packets cannot be lost by applying an error model to downlink only.

As a measure of performance, RDP and RLR explained in Sect. 3.1 were used and we set the coefficients for (7) such that $\alpha=0.0029, \beta=1.0304$, $\gamma=0.000583$. These numbers were obtained through least-square-error model fitting of the various experiments. All the other parameters of RUDP, except the maximum number of retransmission, were set as recommended in [7.

The performance of the proposed UER scheme was compared with the equal error recovery (EER) scheme where the maximum number of retransmission of $3(N=3)$ was applied to all levels of the ALM tree. Delay constraint, $D_{\text {total }}$, was set as the RDP value from the EER with $N=3$, meaning that we want to improve the error recovery performance of the UER under the same delay penalty condition as in the EER scheme. The optimal values of the maximum number of retransmission at each level were obtained from (17) and represented in Table 1.

Relative delay penalties of the proposed UER and the EER scheme are compared in Table 2. It can be seen from the table that the RDP values of the UER are almost same as the ones of the EER. This is simply because the UER is designed assuming the delay constraint of the EER. We can also see from the

Table 1. Optimal values of the maximum number of retransmission at each level

\begin{tabular}{cccccccccc}
\hline $\begin{array}{c}\text { The number of links } \\
\text { from source to leaf }\end{array}$ & 0 & 1 & 2 & 3 & 4 & 5 & 6 & 7 & 8 \\
\hline 5 & 5 & 4 & 3 & 2 & 2 & - & - & - & - \\
6 & 5 & 4 & 3 & 2 & 2 & 1 & - & - & - \\
7 & 6 & 4 & 3 & 3 & 2 & 2 & 1 & - & - \\
8 & 6 & 5 & 4 & 3 & 2 & 2 & 1 & 1 & - \\
9 & 6 & 5 & 4 & 3 & 3 & 2 & 2 & 1 & 1 \\
\hline
\end{tabular}


Table 2. Comparison of relative delay penalty

\begin{tabular}{ccc}
\hline The number of hosts (levels) & UER & EER \\
\hline $63(6)$ & 5.39259 & 5.393329 \\
$127(7)$ & 6.424505 & 6.431873 \\
$255(8)$ & 7.488069 & 7.47746 \\
$511(9)$ & 8.592417 & 8.583579 \\
$1023(10)$ & 9.694796 & 9.686914 \\
\hline
\end{tabular}

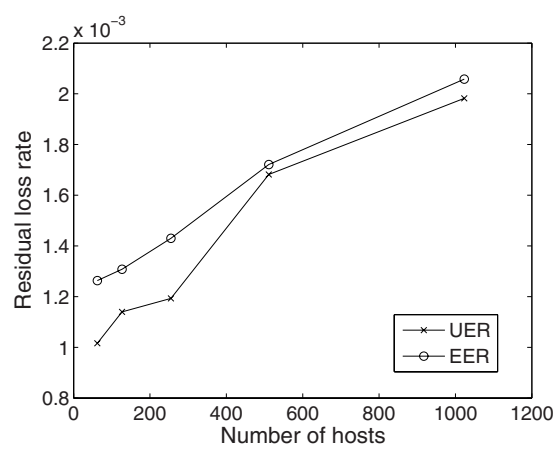

(a) Residual loss rate

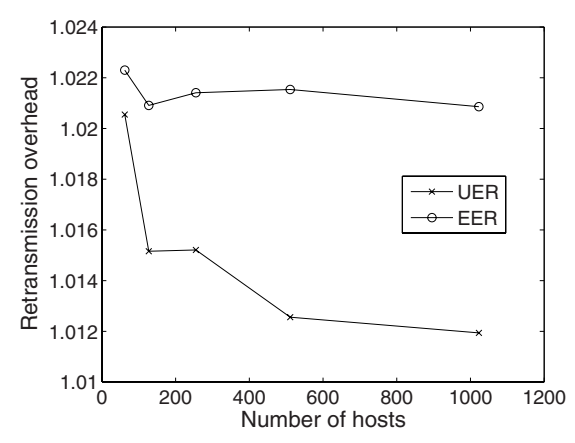

(b) Retransmission overhead

Fig. 3. UER performance versus number of hosts

table that the increment of the delay using RUDP is acceptable for streaming application. The increment of delay penalty is less than $10 \%$ compared to the error-free environments.

The residual loss rate versus the number of hosts was also compared between the UER and the EER and it is shown in Fig. 3(a). As the plot shows, the performance of the UER provides better residual loss rate compared to that of the EER. However, the performance gain of the UER is not perfectly linearly dependent on the number of hosts (there is a dip near 255 hosts). This is because the residual loss rate of UER strongly relies on the distribution of high packet loss rate link. The performance gain of the UER will be higher if the network paths near the source node have high packet loss rates.

In addition to the two performance measures (RDP, RLR) that we discussed in Sect. 3.1, we also analyzed the retransmission overhead of each error recovery scheme as shown in Fig. 3(b). Retransmission overhead represents the ratio of the number of all packets traversing a physical link to the number of source packets in the ALM tree. As seen from the Fig. 3(b) the retransmission overhead of the UER is much smaller than that of the EER. This is because the UER uses smaller value for the number of maximum retransmission in the lower part of the ALM tree and the lower part has much more number of links connected than its higher part. This also explains why the retransmission overhead becomes smaller as the number of host increases. 
It can also be inferred from Fig. 3(a) and Table 2 that error recovery performance and delay characteristic of RUDP is good enough to be applicable to any real-time streaming application based on the UDP. Optimization for dedicated streaming application is also very easy due to its simplicity and flexibility.

\section{Conclusion}

In this paper, we proposed and investigated an unequal error recovery (UER) scheme to recover packet loss in application-level multicast (ALM). The UER was designed to make use of the hierarchical structure of the ALM tree and showed good performance results. As an error recovery methodology, the retransmissionbased RUDP was used on top of the UDP. The performance of RUDP is heavily dependent on the maximum number of retransmission so that we modeled the relative delay penalty and the residual loss rate using this parameter and optimal value was obtained using Lagrange theory. The simulation results demonstrated the benefit of the proposed UER approach. Under acceptable delay penalty, residual loss rate can be improved up to $10 \%$, compared to the baseline error recovery scheme which has equal performance in all levels of the ALM tree.

\section{References}

1. Ganjam, A., Zhang, H.: Internet Multicast Video Delivery. Proc. IEEE 93 (2005) 159-170

2. Yiu, W.-P.K., Wong, K.F.S., Chan, S.-H.G., Wong, W.C., Zhang, Q., Zhu W.W., Zhang, Y.Q.: Lateral Error Recovery for Media Streaming in Application-Level Multicast. IEEE Trans. Multimedia 8 (2006) 219-232

3. Tan, W.-T., Zakhor, A.: Video Multicast Using Layered FEC and Scalable Compression. IEEE Trans. Circuits Syst. Video Technol. 11 (2001) 373-386

4. Noguchi, T., Yamamoto, M., Ikeda, H.: Reliable Multicast Protocol Applied Local FEC. Proc. IEEE ICC 8 (2001) 2348-2353

5. Towsley, D., Kurose, J., Pingali, S.: A Comparison of Sender-Initiated and ReceiverInitiated Reliable Multicast Protocols. IEEE J. Sel. Areas Commun. 15 (1997) 398-406

6. Pierre, D.A.: Optimization Theory with Applications. New York:Dover (1986)

7. Bova, T., Krivoruchka, T.: Reliable UDP Protocol. Internet Draft, Network Working Group [OnLine]. Available: draft-ietf-sigtran-reliable-udp-00.txt

8. NS-2: network simulator [OnLine].http://www.isi.edu/nsnam/ns 